\title{
Relational Ethics and Nurses-Client Relationship in Nursing Practice: Literature Review
}

\section{Upasen $R^{*}$}

Faculty of Nursing, Chulalongkorn University, Thailand

*Corresponding author: RatchaneekornUpasen, Faculty of Nursing, Chulalongkorn University, Borommaratchachonnanisrisataphat Building, the $11^{\text {th }}$ floor, Rama I road, Bangkok, 10330, Thailand, Tel: +6683-776-2828; Email: Upasen.r@gmail.com

\section{Review Article \\ Volume 1 Issue 1}

Received Date: July 11, 2017

Published Date: July 29, 2017

DOI: $10.23880 / \mathrm{mhrij}-16000102$

\section{Abstract}

Background: This review focuses on exploring the concept of the nurse-client relationship as it may be informed by relational ethics. Relational ethics is a new approach to ethical practice in health care and can be a framework for nurses and other health professionals in considering how to help patients and families.

Purpose: To review the basic elements of relational ethics prior to summarizing the existing literature related to the nurse-client relationship in psychiatric and mental health settings.

Methods: Qualitative studies and other research were identified by using the PubMed, CINAHL, ScienceDirect, Academic search databases, and by hand search in the library. The search limited to the period 2000-2016. The exception to this is the inclusions of some articles that are considered as classic in this area of nurse-client relationship and ethics.

Results: 45 studies that met the final inclusion criteria were selected to analyze theme. The argument is then made that relational ethics can inform therapeutic nurse-client relations in important ways and that it constitutes important knowledge for psychiatric and mental health nurses. The existing literature, however, does not identify strategies that support the practice of relational ethics in this way. This article described how relational ethics is a powerful guide to ethical and effective nursing practice.

Conclusion and Recommendation: There is a growing need to better understand relational ethics within the nurseclient relationship in order to identify ways to support encountered situations.

Keywords: Relational Ethics; Ethics; Mental Health; Nursing Practice; Relationship; Therapeutic

\section{Introduction}

Psychiatric nursing practice focuses on interpersonal relationships for providing care for people with mental and behavioral disorders. Interpersonal relationship is an important paradigm in psychiatric nursing [1]. It aims to promote and maintain the behaviors that lead to a client's healthy life [2,3]. The nurse-client relationship is considered the basis of psychiatric care. It is an interpersonal process that is developed over time between nurses and their clients. The concept of the nurse-client relationship is grounded in the work of 


\section{Mental Health \& Human Resilience International Journal}

Peplau [4]. It was expanded by a variety of nurse theorists, such as King (1990), Paterson and Zderad (1988), and Hagerty and Patusky, (2003) [5-7]. Psychiatric nurses often apply the notion of interpersonal relationship when giving care to their clients. In addition to their central place is psychiatric nursing, Peplau's, King's, and Paterson and Zderad's theories have been taken up as conceptual frameworks across other nursing practice areas as well. The theories address the process or phase of the nurse-client relationship for helping clients. For instance, Peplau identified four phases of the nurseclient relationship: orientation, identification, exploitation, and the resolution phase [8,9]. Thus, the nurse-client relationship can be the "heart, soul and essence of psychiatric nursing" and psychiatric nurses have been the beneficiary of Peplau's interpretation [1]. Within the nurse-client relationship, there is a space where relational ethics can be placed. This therapeutic relationship can be understood as a relational space where the ethics of the relationship is shaped [10].

Relational ethics is defined as an action ethic that is placed within the interpersonal relationship. The action ethics include engagement, mutual respect, embodiment, and interdependent environment $[11,12]$. The relational ethics is a new approach to ethical practice in health care. Moreover, relational ethics may be another framework for nurses and other health professionals in considering how to help patients and families $[13,14]$. For example, a study found that relational ethics help nurses acknowledge the importance of opening dialogue, considering a diversity of perspectives, and understanding the need for attention to environment in order to provide appropriate health care [13]. Psychiatric nurses need to be understood how the relational ethics is and how to related to nurse-client relationship.In this article, the authors wish to introduce the concept of relational ethics as a powerful tool when thinking about the nurse-client relationship and how to best care for clients with mental health problems and/or disorders. This article aims to understand the meaning and core concept of relational ethics and nurse-client relationship.

\section{Review of the Literature Addressing Relational Ethics and Nursing Care}

The core elements of relational ethics are highly congruent with those of the nurse-client relationship. I searched for studies that used relational ethics as a conceptual framework in caring for clients and working with family members in order to analyze themes relevant to nurse-client relationship and relational ethics.Qualitative studies and other research were identified by using the PubMed, CINAHL, ScienceDirect, Academic search databases, and by hand search in the library. The search limited to the period 2000-2016. The exception to this is the inclusions of some articles that are considered as classic in this area of nurse-client relationship and ethics. Number of studies for review in total was 409 studies and that met first inclusion criteria were collected. The first inclusion criteria were: 1) Studies were qualitative or other designs. 2) Studies were required to address about ethic in health care. 3) Studies were explained health care providers and client relationship. 4) Studies had to be presented in English.Key words that were used to search in the literature are nurse-client relationship, relational ethics, interpersonal relationship, ethical relationship, psychiatric nursing, nursing ethics, and ethic of care. The articles obtained in full text were selected in the literature. Then the 45 studies that met the final inclusion criteria were selected to analyze theme. The final inclusion criteria of the studies consist of: 1) Studies were required to explain relational ethics and ethical issues in nursing practice 2) Studies were required to explain nurse-client relationship.

\section{Findings}

The findings were organized to five themes: meaning of relational ethics, core elements of relational ethics, nurseclient relationship: a powerful tool in psychiatric nursing, the Nurse-client relationship and relational ethics, and relational ethics: a powerful guide to ethical nursing practice. Summary of major themes and sub- themes were described in Table 1 as follows:

\begin{tabular}{|l|l|}
\hline \multicolumn{1}{|c|}{ Major themes } & \multicolumn{1}{c|}{ Sub-themes } \\
\hline \multirow{4}{*}{ Understanding relational ethics } & Core elements of relational ethics \\
\cline { 2 - 2 } & - Engagement \\
\cline { 2 - 2 } & - Mutual respect \\
\cline { 2 - 2 } & - Embodiment \\
\cline { 2 - 2 } & - Interdependent environment \\
\hline \multirow{2}{*}{ Nurse-client relationship: a powerful tool in } & - A tool to create a therapeutic relationship \\
\hline
\end{tabular}

Upasen R. Relational Ethics and Nurses-Client Relationship in Nursing Practice: Literature Review. Ment Health Hum Resilience Int J 2017, 1(1): 


\section{Mental Health \& Human Resilience International Journal}

\begin{tabular}{|c|c|}
\hline \multirow[t]{3}{*}{ psychiatric nursing } & - A process for engaging clients and providing nursing interventions. \\
\hline & - A model for sharing experience and coping better \\
\hline & $\begin{array}{l}\text { - An important way to assess client's needs, plan and implement } \\
\text { interventions, and evaluate }\end{array}$ \\
\hline \multirow{3}{*}{ Nurse - client relationship and relational ethics } & - A relational space where the ethics of the relationship is shaped \\
\hline & $\begin{array}{l}\text { - Mutual relating is built within a relationship of trust and mutual } \\
\text { respect }\end{array}$ \\
\hline & - Relational ethics evoke a sense of trust in the relationship \\
\hline \multirow{3}{*}{$\begin{array}{c}\text { Relational ethics: a powerful guide to ethical } \\
\text { nursing practice }\end{array}$} & $\begin{array}{l}\text { - A framework to explore human experiences and to understand } \\
\text { clinical phenomena }\end{array}$ \\
\hline & $\begin{array}{l}\text { - A concrete ethical model for health professional to response the } \\
\text { different ethical issues }\end{array}$ \\
\hline & - A framework in providing quality care for the patients. \\
\hline
\end{tabular}

Table 1: Major themes and sub-theme of relational ethics and nurse-client relationship.

\section{Understanding Relational Ethics}

Relational ethic is defined as an "action ethic" [11] that explicitly situates ethics in relationship. Relational ethics is a relatively new perspective on nursing care. It situates ethics within a context of human relations that emphasizes human interdependency and reciprocity within which personal autonomy is embedded. Relational ethics requires health care providers base ethical actions on relations and commitments to patients, families, and others [12]. In Canada, the Relational Ethic Project was developed by VangieBergum (a nurse) and John Dossetor (a physician). The framework arose out of research conducted at University of Alberta, Canada (1993-2001). The project explored the ethical commitments enacted in everyday health care situations. Core elements of a relational ethics are described as engagement, mutual respect, embodiment, and interdependent environment and were described in the text, Relational Ethics: The Full Meaning of Respect $[15,12]$. The elements of relational ethics will be described as follow:

\section{Core Elements of Relational Ethics}

Engagement is about human to human connection. It is situated in the commitment between individuals. Ethical professional practice requires understanding the other's situation, perspective, and vulnerability [15-18]. An opening question when nurses and family members form a relationship is "How do we engage with each other?" [12]. Looking at a person as a whole, appropriate vulnerability, conversation, and time management are aspects of engagement. Engagement is not only the mechanistic behavioral processes of communication such as active listening, eye contact, and touch, but also a capacity to relate and be with one another. In addition, opening a space for listening to other people's expression of feelings, perspectives, and concerns/needs is needed [19]. If appropriate engagement is possible, underengagement and over-engagement will not occur [12]. A relationship can be reconstructed by an action ethics where people connect with other and have mutual respect.

Mutual respect involves self-respect and respect for others and from others. The respect for someone is not always easily achieved. It may require engaging with different attitudes, genders, experiences, knowledge, beliefs, culture, power, etc. If the care provider's perspective differs from the other person's viewpoint, mutual respect can be difficult [12]. Expression of respect for nurses who work with patients and families requires reflection of self-understanding and understanding of others. Mutual respect is reciprocal: it cannot occur if nurses work without patients or family members' understanding, interdependence, personhood, andconnection, in particularly acknowledgement the patients as the persons $[20,21]$. If health care professionals and patients/family members can establish mutual respect, the mutual respect leads to mutual power and re- empowerment. The connection between body and mind and objectivity and subjectivity should be integrated and respected in a relationship. 


\section{Mental Health \& Human Resilience International Journal}

Embodiment, in relational ethics, refers to the role emotion play in our ethical decisions and actions. Relationships require both objective and subjective knowledge. That means that relationships cannot occur without embodiment. Our body is the means by which we experience the world [22]. It is impossible to separate our mind from our body [23]. Embodied knowledge focuses on healing the division between body and mind, self and others, and objective and subjective. Within relationships, we will find a relational space between body and mind, objective and subjective, and self and others are found. The relational space in a relationship needs attention to inter subjectivity where body and mind cannot be separated [12]. In nursing practice, nurses will provide holistic care for the patients, including for patients' bodies, minds, and spiritual dimensions as well as their environments.

Interdependent environment, the practice of relational ethics requires recognizing that we exist in an interdependent environment. The environment is defined as the circumstances, objects, or conditions by which one is surrounded. Health professionals need to attend to the environments of individuals, including the health care system, society at large, and local communities. Ethical practice cannot occur without personal, social, or political elements. Supportive environments not only help people who are vulnerable to cope better, but also increase their ability to related to other people [11]. This type of environment requires giving freedom and choice for making decisions, inclusive of individual and social choice. The context of care should be always considered in clinical practice [12]. For example, in providing care for terminal ill patients, there are many factors that influence or are significant to health care providers' caring, such as families, relationshipswith physicians, and restrictions of the unit or organization. The health care providers should attend particularly to the environment where there may be high incidents of adverse outcomes (Leung \& Esplen, 2008) [21].

\section{Nurse-Client Relationship: A Powerful Tool in Psychiatric Nursing}

The nurse-client relationship is a key element in the nursing process [2]. It is an important way in which nurses are able to assess accurately client's needs, diagnose clients, help clients establish outcomes, plan and implement interventions, and evaluate the effectiveness of nursing process [4]. In psychiatric nursing, practitioners often interact with people who are experiencing mental health problems or disorders and are in need of mental health services. It is a challenge for nurses and requires them to have the skills, knowledge, and perception to think carefully about what will be the best for and most helpful to clients $[24,25]$. At various points during their relationship with a client, a psychiatric nurse's role may encompass being an educator, advocate, counselor, teacher, or socializing agent [8,26-28]. Moreover, the nurses use themselves as a tool to create a therapeutic relationship with clients to help them to grow, change, and heal [29]. Peplau (1991) [8] suggests that nurses must have self-understanding to promote a client's growth and to avoid limiting a client's choices. Wright (1994) [30] explains further that nurses are expected to use their own feeling responses in a professional way, in order to better understanding of client's emotion and needs, which lead to providing appropriate and therapeutic care. This "therapeutic use of self" is a core skill of psychiatric and mental health nursing practice [31]. In addition, the interpersonal dimension of the nurse-client relationship can be the vehicle for implementing the nursing process. Peplau (1991) [8] shows this well in her work on the nurse-client relationship, Interpersonal relations in Nursing: $A$ conceptual frame of reference for psychodynamic nursing. Her theory guides nurse practitioners in deepening their understanding of interpersonal relations in nursing situations. Similarly, King's (1990) [5,32] nursing theory in which the nurse-client relationship is perceived as a learning experience where two people interact to face health problems, share, discover ways to support the situation, and resolve the problems, further enriches the role of interpersonal relations in nursing practice.

Several studies have explored the effectiveness of the nurse-client relationship [29-33]. This includes a study in forensic psychiatric nursing, a setting in which trust between client and nurse is made more complex by security demands [16], that indicated nurses recognized the nurse-patient relationship as a powerful tool. These researchers found, as well, that oral communication was significantly more powerful than written communication with patients [33]. In another study, Eby \& Brown (2005)[28] found that nurses learn from this process how to engage with clients and how to provide appropriate nursing interventions.

Some studies focus on the process of developing interpersonal relationships in psychiatric nursing $[34,35]$. For instance, Hanson \& Taylor (2000) [34] used a model of the mental health nurse-client relationship based on Martin Buber's concepts of the I-Thou (Being-with) and IIt (Doing-with) relationship. They found that the model is very useful for community mental health nurses in 


\section{Mental Health \& Human Resilience International Journal}

helping persons with mental illness in the community. The framing of the relationship in Buber's model fosters the sharing of client experiences with nurses which in turn allows nurses to help clients to cope better and to live more effectively in the world.

\section{The Nurse-Client Relationship and Relational Ethics}

Nurse-client relationship is a therapeutic relationship that encompasses the tool of therapeutic use of self. It is goaldirected. That means that the nurse and client decide together what the goal of the relationship will be. The objectives of the relationship are focused on learning, growth promotion, and problem solving. Essentially, it is an effort to promote change in some aspect of the client's life. The nurse and client determine together how much time they are able to spend. Nurses need to provide information based on facts and without bias and personal opinion Moreover, it is the client's feelings and opinions that are significant and, thus, the nurse must work to foster an atmosphere of mutual respect and mutual understanding to facilitate the client's comfort in sharing $[26,27,36]$. This atmosphere that evolves through the therapeutic relationship can be understood as a space, a relational space where the ethics of the relationship is shaped [10]. In addition, their findings indicate that some parts of the interaction between health care providers and clients/families reflect an element of relational ethics, that of mutual respect. Mutual respect occurs when people connect meaningfully with others. It includes self-respect and respect for others and from others. With mutual respect, power is understood as predominately "powerwith", not "power-over" [12]. One such study was McCann's and Baker's (2001) [35] research into mutual relating, a model of interpersonal relationships (between nurses and family members) in the community context. They found that many strategies are related to mutual relating, including attempting to understand, being friendly, turning in, revealing oneself, being there for clients, and maintaining confidentiality. They also found that mutual relating is built within a relationship of trust and mutual respect. This research suggests that mutual respect, a core element of relational ethics, is essential to therapeutic relationships.

Other studies reveal the presence of several core elements of a relational ethics, including mutual respect, engagement, and attention to the environment (or context) in the nurse-client relationship. These elements offer a basis for considering ethical practice. Nurses may use these elements to inform their care, including how to respond in crisis situations [15]. Austin and colleagues
(2009) [37] conducted a participatory action research study to examine the relationships between families of residents of traditional continuing care facilities and the health care team. In one of the findings, the researchers found that mutual respect was shaped through the interaction between health care team and families of the residents. The mutual respect was evidenced in the knowing one another's names and the acknowledging of the need to understand another's perspectives. The researchers also explicitly noted that mutual respect is a basis element of ethical relationships. In addition, genuine engagement and the interdependence of family and health care team were key to enable the residents to gain or maintain quality of life. The findings indicate further that relational ethics is important to therapeutic interpersonal relationships.

Marcellus (2005) [38] found that four themes of relational ethics appeared within the relationship between public health nurses and families: mutual respect, engaged interaction, embodiment, and creating environment. These four themes appeared to evoke a sense of trust in the relationship. Considering explicitly the ethical foundations of therapeutic relationships may guide public health nurses in their work with families. Another study by Gladstone and Wexler (2000) $[39,40]$ found that a collaborative relationship between staff and family members can have ethical dimensions: open communication, attentive behavior, and display empathy towards each other. Families' work with staff is interdependent, they share responsibilities, and they share decision making. The communication between staff and family members is characterized by two-way communication. This finding indicates that staff and family members were mutually respectful and tried to engage each other's concerns by sharing their own perspectives.

\section{Relational Ethics: A Powerful Guide to Ethical Nursing Practice}

Relational ethics is a powerful guide to ethical and effective nursing practice. Studies have used relational ethics as a framework to explore human experiences and to understand clinical phenomena [11,15,20,37-42]. Relational ethics help nurses recognize the value of opening dialogue, considering a diversity of perspectives, and understanding the need for attention to environments in order to provide therapeutic care $[13,15]$. In a study conducted by Galvin (2010) [42], critical care nurses were found to place clients at the center of critical care. The critical care nurses provided critical care with a balance of hand, head, and heart. 


\section{Mental Health \& Human Resilience International Journal}

"Hand" is defined as technical skills of nurses. "Head" is defined as protocols and evidences that were employed by nurses. "Heart" is defined as ethical and human dimensions that are acknowledged by nurses. Galvin (2010) [42] also suggests that nurses' specialized knowledge, expertise care, and the capacity of caring through openheartedness and embodied relational understanding can guide the critical care nursing to be completed and meet optimal nursing outcomes. Moreover, the relational ethics can be a concrete ethical model for health professional to response the different ethical issues in health care practice and research $[43,44]$.

In another study in the mental health field by Austin, Bergum\&Nuttgens (2004) [20], "Addressing oppression in psychiatric care: a relational ethics perspective," it was suggested that, in providing care for patients with mental illness,practitioners focused on using protocols and procedural guidelines. Such protocols and procedures were too often insufficient in addressing systemic problems in psychiatric practice. Relational ethics was offered as a better way to address the issue of oppression in psychiatric care and to practice effectively and ethically in caring for persons made vulnerable by mental health problems and disorders.

In general fields, there are studies that reported that relational ethics is a new way to help health professionals resolve health problems of clients. Evans, Bergum, Bamforth and MacPhail (2004) [10] examined relational ethics and genetic counseling. The goal of their study was to explore the realities of genetic counseling in order to identify effective ways to counsel clients, as well as to consider how relational ethics might enhance counseling effectiveness [45-48]. The researchers found the three aspects of relational ethics -engagement, dialogue, and presence - that can improve quality care and the outcome of genetic counseling. For instance, many of the participants perceived value in the friendliness of counselors; dialogue was seen as a connection between clients and counselors; and "presence" helped clients to connect with and share their experiences with someone.

In a study conducted by Sellevold, Egede-Nissen, Jakobsen \& Sorlie (2013) [41], researchers explored healthcare providers' experience with quality care and described the healthcare providers' experience with ethical challenges. Relational ethics was also used as the framework for the study. The researchers found three themes; healthcare providers experienced quality care when they emphasize the patient'sresponse by accepting the patients as an individual and sentient awareness; when they understand the patient's expression, in particular emotion and bodily expression; and reciprocity in expression is based on the patient's bodily responseand recognition by the patient. These findings reflect the importance of using relational ethics as a framework in providing quality care for the patients.

The literature shows that relational ethics is a new way to conceive of ethical practice. It can be a basic concept for health practitioners and help them to consider how to act ethically in complex and critical care situations. Psychiatric nurses and others can use relational ethics to guide the development of their relationships with persons with health problems and their families. The authors believe that if psychiatric nurses or general nurses emphasize relational ethics through the interpersonal relationship processes, patients and their families will be effectively supported when dealing with serious health problems.

\section{Implications and Conclusion}

In short, relational ethics is an action ethic that arises during the relationship. It is a new way to conceive of ethical practice with clients and their families. Attending to the core elements of mutual respect, engagement, embodiment and interdependent environment can augment the skills psychiatric nurses currently use to establish therapeutic relationships with clients, as well as foster ethical practice.

Relationship is an important paradigm in nursing practice. The nature of the relationships is established through the process of interaction. It is a helping relationship that is shaped by nurses and clients and an effective tool in providing care for individuals with mental illness and their families. The nurse-client relationship manifests in various forms. Nurses integrate skills and knowledge in providing care for client through the process of the nurse-client relationship: orientation, identification, exploitation, and resolution phase. They are enable by this relationship provide effective nursing interventions for the clients and their families. There are some studies that show the powerful or effective ways of the nurse-client relationship and relational ethics. However, there is little research in nursing practice on relational ethics. The further research is needed.

\section{Acknowledgement}

I wish to acknowledge the contributions to conduct this article by Faculty of Nursing, University of Alberta and 


\section{Mental Health \& Human Resilience International Journal}

Faculty of Nursing, Chulalongkorn University for supporting time and all material resources.

\section{Conflict of Interest Statement}

The author declares that there is no conflict of interest in this study.

\section{References}

1. Raingruber B (2003) Nurture: The fundamental significance of relationship as a Paradigm for mental health nursing. Perspective in Psychiatric care 39(3): 104-135.

2. Arnold E (2007) Professional Guide to Action in Interpersonal Relationship. In: E Arnold \& K U Boggs (eds), Interpersonal Relationships. Missouri: SAUNDERS, pp: 66-78.

3. Yunibhand J (1999) Nursing for Health Life. Journal of Psychiatric Nursing and Mental Health 13: 1-11.

4. Fontaine KL (2003) Mental Health Nursing. Pearson Education, Inc, New Jersey.

5. King IM (1990) King's Conceptual Framework and Theory of Goal Attainment. In: ME Parker (eds), Nursing Theories in Practice.National League for Nursing, New York.

6. Paterson JG, Zderad LT (1988) Humanistic nursing. National League for Nursing, New York.

7. Hagerty BM, Patusky KL (2003) Reconceptualizing the Nurse-Patient Relationship. Journal of Nursing Scholarship 35(2): 145-150.

8. Peplau HE (1991) Interpersonal Relations in Nursing. Springer Publishing Company, New York.

9. Peplau HE (1995) Some unresolved issues in the era of biopsychosocial nursing. Journal of the American Psychiatric Nurses Association 1(3): 92-96.

10. Bergum V (2004) Relational ethics in nursing. In: JL Storch, P Rodney \& R Starzomski (Eds), Toward a moral horizon: Nursing ethics for leadership and practice. Pearson Prentice Hall, Toronto.

11. Evans M, Bergum V, Bamforth S, MacPhail S (2004) Relational Ethics and Genetic Counseling. Nursing Ethics 11(5): 459-471.
12. Bergum V, Dossetor J (2005) Relational Ethics: The full Meaning of Respect. Hagerstown MD, University Publishing.

13. Austin W (2008) Ethics in a time of contagion: A relational perspective. Canadian Journal of Nursing Research 40(4): 10-24.

14. Austin W, Goble E, Kelecevic J (2009) The ethics of forensic psychiatry: Moving beyond principles to a relational ethics approach. Journal of Forensic Psychiatry \&Psychology 20(6): 835-850.

15. Austin W (2001) Relational Ethics in ForensicPsychiatric Settings. Journal of Psychosocial Nursing \& Mental Health Services 39(9): 12- 17.

16. Austin W, Bergum V, Dossetor J (2003) Relational ethics: An action ethic as a foundation for health care. In: V Tschudin (Eds), Approaches to Ethics. Nursing Beyond Boundaries, Edinburgh: Butterworth Heinemannn, p: 45-51.

17. Austin W, Bergum V, Goldberg L (2003) Unable to answer the call of our patients: mental health nurses' experience of moral distress. Nursing Inquiry 10(3): 177-183.

18. Austin W, Nuttgens S, Bergum V, Peternelj-Taylor C (2006) A Re-visioning of Boundaries in Professional Helping Relationships: Exploring other Metaphors. Ethics \& Behavior 6(2): 77-94.

19. Wright D, Brajtman S (2011) Relational and embodied knowing: Nursing ethics within the interprofessional team. Nurs Ethics 18(1): 20-30.

20. Austin W, Bergum V, Nuttgens S (2004) Addressing oppression in psychiatric care: A relational ethics perspective. Ethical Human Psychology and Psychiatry 6(1): 69-78.

21. Leung D, Esplen MJ (2008) Alleviating existential distress of cancer patients: can relational ethics guide clinicians?. European Journal of Cancer Care 19(1): 30-38.

22. Merleau-Ponty (1962) Phenomenology of Perception. Routledge Classics, New York.

23. Matthews M (2002) The philosophy of merleauponty. Bucks: Acumen. 


\section{Mental Health \& Human Resilience International Journal}

24. Deering C, Frederick J (2003) Therapeutic relationships and communication. In: $\mathrm{W} \mathrm{K}$ Mohr (Eds), Psychiatric Mental Health Nursing. Lippincott Williams \& Wilkins, Philadelphia.

25. Deering C (2006) Therapeutic relationships and communication. In: WK Mohr (Eds), Psychiatric Mental Health Nursing. Lippincott Williams \& Wilkins, Philadelphia.

26. Townsend CM (1999) Essential of Psychiatric/Mental Health Nursing. FA Davis Company, Philadephia.

27. Townsend CM (2000) Psychiatric mental health nursing concepts of care. FA Davis Company, Philadephia.

28. Eby L, Brown N (2005) Mental Health Nursing Care. Pearson Education Inc, New Jersey.

29. Videbeck S (2001) Psychiatric Mental Health Nursing. Lippincott Williams \& Wilkins, Philadelphia.

30. Wright $H$ (1994) The therapeutic relationship. In: H Wright \& M Giddey(Eds), Mental Health Nursing. Chapman \& Hall, London.

31. Uys LR (1980) Toward the development of an operational definition of the concept of "therapeutic use of self". International Journal of Nursing Studies 17(3): 175-180.

32. Morris M (2009) Mental health for primary care: A practical guide for non-specialists. Rackliffe Publishing, Oxford.

33. Martin T, Street AF (2003) Exploring evidence of the therapeutic relationship in Forensic psychiatric nursing. Journal of Psychiatric and mental Health Nursing 10(5): 543-551.

34. Hanson B, Taylor MF (2000) Being with, Doing with: a model of the nurse-client relationship in mental health nursing. Journal of Psychiatric and Mental Health Nursing 7(5): 417-423.

35. McCann TV, Baker H (2001) Mutual relating: developing interpersonal relationships in the community. J Adv Nurs 34(4): 530-537.

36. Forchuk C (1993) Hildegarde E Peplau: Interpersonal Nursing Theory. SAGE publications Inc, California.
37. Austin W, Goble E, Strang V, Mitchell A, Thompson E, et al. (2009) Supporting Relationships between Family and staff in continuing Care Settings. Journal of Family Nursing 15(3): 360-383.

38. Marcellus L (2005) The ethics of relation: public health nurses and child protection clients. Journal of Advanced Nursing 51(4): 414-420.

39. Gladstone J, Wexler E (2000) A family perspective of family/ staff interaction in long - term care facilities. Geriatric Nursing 21(1): 16-19.

40. Gladstone J, Wexler E (2002) Exploring the relationships between families and staff caring for residents in long - term care facilities: Primary caregivers' perspective. Canadian Journal on Aging 21(1): 39-46.

41. Sellevold GS, Egede-Nissen V, Jakobsen R, Sorlie V (2013) Quality care for persons experiencing dementia: The significance of relational ethics. Nursing Ethics 20(3): 263-272.

42. Galvin KT (2010) Revisiting caring science: some integrative ideas for the "head, hand and heart" of critical care nursing. British Association of Critical Care Nurses 15(4): 168-175.

43. Barbosa A (2011) Relational ethics and psychiatry. European Psychiatry 26: 753-763.

44. Lange R, Williams AS (2011) Linking adults' problems with children's pain: Legal, Ethical and Clinical issues. Psychiatry Psychology and Law 18(2): 232-239.

45. Macdonald H (2007) Relational ethics and advocacy in nursing: literature review. J Adv Nurs 57(2): 119126.

46. Shaw E (2011) Relational Ethics and Moral Imagination in Contemporary Systemic Practice. The Australian and New Zealand Journal of Family Therapy 32(1): 1-14.

47. Stevenson C, Grieves M, Stein-Parbury J (2004) Patient and person: Empowering interpersonal relationships in nursing. Elsevier Ltd, China.

48. Varcarolis EM, Carson VB, Shoemaker NC (2006) Foundations of Psychiatric Mental Health Nursing. Missouri: SAUNDERS, pp: 834. 


\section{Mental Health \& Human Resilience International Journal}

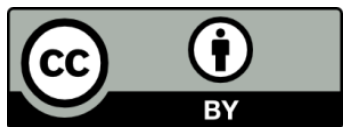

